\title{
An investigation into the development of right displaced abomasum by rolling 268 dairy cows with left displaced abomasum
}

\author{
J. V. González-Martín,, ${ }^{1,2}$ N. Pérez-Villalobos, ${ }^{1,3}$ W. Baumgartner, ${ }^{4}$ and S. Astiz ${ }^{5 *}$ \\ ${ }^{1}$ Department of Animal Medicine and Surgery, Universidad Complutense de Madrid (UCM), 28040 Spain \\ ${ }^{2}$ Trialvet Veterinary Consultancy and Research SL, 28721 Madrid, Spain \\ ${ }^{3}$ Faculty of Biomedical Sciences, Universidad Europea de Madrid, 28670 Spain \\ ${ }^{4}$ University of Veterinary Medicine, University Clinic for Ruminants, 1210 Vienna, Austria \\ ${ }^{5}$ Department of Animal Reproduction (INIA), UCM, 28040 Spain
}

\section{ABSTRACT}

Right displaced abomasum (RDA) and abomasal volvulus (AV) are common diseases in cattle. However, presently there is no consensus regarding the pathogenesis and nomenclature of the different positions that the abomasum can adopt in the right side of the cow. Therefore, the objective of this study was to describe the etiopathogenesis of the right displaced abomasum according to the description of the features observed in 268 cows with left displaced abomasum (LDA), which were rolled to induce the movement of the abomasum from the left side to the right (in fact, inducing an RDA in $44 \%$ of cases), to facilitate the surgical approach, and immediately surgically treated (abomasal omentopexy from the right side). The observed RDA positions matched previously published descriptions and names, and the authors propose a holistic and simpler nomenclature and a clear hypothesis on RDA etiopathogenesis. In $54.1 \%$ of the cases, the abomasum moved to a normal position after rolling. The most common RDA position observed was caudal displacement of the RDA (CdRDA; 70 out of 119 RDA cases; $26.1 \%$ of the total number of cows), followed by lateral displacement (LRDA; 32 of 119; $11.9 \%$ of all cases), cranial displacement (CrRDA; 8 of $119 ; 3 \%$ of the total), medial displacement (MRDA; 4 of $119 ; 1.5 \%$ of the total), and finally, displacement lateral to the omasum (LORDA; 3 of 119; $1.1 \%$ of the total). Adverse events directly due to the rolling procedure were mesenteric root volvulus (3 of 268), cecocolic volvulus (2 of 268) and torsion (1 of 268), and uterus torsion (2 of 10 pregnant cows); 1 abortion (1 of 10 pregnant cows) was also observed. We suggest that CdRDA is the first stage of an RDA that can become an LRDA with increasing pressure. An LRDA can become an AV if rotating coun-

Received February 22, 2019.

Accepted August 6, 2019.

*Corresponding author: astiz.susana@inia.es terclockwise, viewed from above. An LDA, CdRDA, or LRDA could be initial forms of LORDA and CrRDA, and occur when forestomachs are empty or when the animal stands up on its front legs first. The LORDA and CrRDA forms can lead to an omasum-abomasum volvulus (OAV) or to a reticulum-omasum-abomasum volvulus (ROAV) when pressure increases. With this study we establish, for the first time, the sequence and incidence of each RDA form and its complications, increasing knowledge of this syndrome and its pathogenesis. This understanding critically aids the surgeon's ability to accurately assess and correct this disease and to predict prognosis.

Key words: abomasum displacement, right, etiopathogenesis, dairy cow

\section{INTRODUCTION}

Although displacements of the abomasum were already known to exist, the first comprehensive study of abomasal displacement in adult cows, including rightside abomasum displacements (RDA), can be read in Dirksen (1961). Since then, studies have continued to improve our understanding of this pathology (Itoh et al., 2017). When considering the pathogenesis of any disease, its forms must be clearly described. In this regard, the study of RDA presents a further challenge (Geishauser, 1995). The description of RDA and abomasal volvulus (AV) forms that achieves the greatest consensus (Constable et al., 2017) is that of Constable et al. (1991), who describe RDA as a mirror image of left displaced abomasum (LDA) with no volvulus and with the abomasal serosal aspect covered or not by the omentum, depending on the nature of the displacement (Hoffsis, 1986; Constable et al., 1991, 2017; Fubini et al., 1991).

Constable et al. (1991, 2017) describe AV as an evolution of RDA that rotates in a clockwise direction (when viewed from the right side), in a vertical plane around a horizontal axis passing transversely across 
the body in the vicinity of the omaso-abomasal orifice and displacing the liver. This has been described as an external method to discriminate AV from RDA, depending on the existence of a "ping" cranial to the 10th rib (AV) or not (RDA; Constable et al., 1991). Another description of AV is that of Trent (2017), who defines it as the abomasum and attached structures rotating in a counterclockwise direction around an axis through the center of the lesser omentum, causing the cranial duodenum to become trapped by the distended abomasal body. The volvulus is usually the result of a $180^{\circ}$ to $270^{\circ}$ rotation of this viscera (Espersen, 1961; Rosenberger, 1994). To enhance clarity, the authors of the current paper use the term "volvulus," because the long axis of the abomasum displaces and twists around its supporting structures (Constable et al., 2017; Trent, 2017). When the term "torsion" is used, it is referring literally to other authors' descriptions.

Omaso-abomasal volvulus (OAV) is described by Constable et al. (1991, 2017), Dirksen (2002), and Gelberg (2017) as a $180^{\circ}$ rotation of the abomasum and omasum around the transverse axis of the lesser omentum, drawing the duodenum cranially, and medial to the omasum. Reticulo-omaso-abomasal volvulus (ROAV) is described by Constable et al. (1991, 2017) and Dirksen (2002) as similar to the OAV, including the reticulum. Besides Constable's classification, 2 additional forms of non-left-sided abomasal displacement have been described: cranial and dorsal to the reticulum displaced abomasum (Habel and Smith, 1981; Fubini, 2007; and Gelberg, 2017); and "right dislocation with a $180^{\circ}$ right torsion" (Espersen, 1961; Rosenberger, 1994), also called medial displacement of the abomasum (Anderson, 2009). See Table 1 for literature references and comparison of definitions of these disorders.

Despite being a very common process in cattle, mechanisms of displacement and gas accumulation in the abomasum remain unclear for the LDA and even more so for the much less frequent RDA (Trent, 2017). Differences in definition, in methods of description, and in the ability of the individual surgeon to correctly identify the location of the abomasal components give rise to a wide range of names and definitions of RDA and AV forms. A more accurate description for this syndrome and its pathogenesis is of more than academic concern, because an understanding of the mechanisms of RDA and AV critically aids the surgeon's ability to accurately assess and correct this disease.

Rolling can be used to study the pathogenesis of abomasum displacement. Toggle pin fixation, along with omentopexy after a right paralumbar fossa laparotomy, are the most common approaches to resolve LDA. In large cows, however, on rare occasions, correction of the LDA can be challenging when performed by the surgeon from the right side of the cow (Trent, 1990). In such cases, rolling can be used to induce movement of the LDA to the right side, to facilitate surgical resolution of the LDA. Omentopexy from the right paralumbar fossa is then performed immediately after rolling. In fact, when this method is used, the LDA is resolved, but an RDA and its very early movements can be induced (St Jean et al., 1989). This feature could be studied scientifically, if all findings after rolling were subsequently recorded. Therefore, given the fact that the different positions and movements of the RDA have not been completely clarified, the objective of this study was to describe the early positioning of the RDA, building an experimental model to approach RDA and $\mathrm{AV}$, with iatrogenically induced RDA after rolling cows suffering LDA.

\section{MATERIALS AND METHODS}

The total subset of 268 LDA-affected cows included in the present study came from a population of about 4,000 Holstein cows from 70 commercial Spanish dairy farms [mean herd size of milking cows $( \pm \mathrm{SD})$ was $75 \pm$ 51 (range of 30 to 300$)$ ], with a mean milk yield ( \pm SD) of approximately $7,638 \pm 1,216 \mathrm{~L}$ (range of 6,000 to $11,000 \mathrm{~L}$ ) per lactation. All herd owners were advised by the same veterinary consulting group. Herds were managed under similar conditions, with ad libitum access to water, and fed twice daily with a TMR that was balanced to meet or exceed nutrient recommendations for lactating dairy cows (NRC, 2001). The TMR consisted primarily of alfalfa silage, corn silage, alfalfa hay, straw, corn, soybean brewer's grain, beetroot pulp, and cotton and soybean hulls, with bicarbonate and corrector salts, with minimal variations depending on the available raw material.

From January 1999 to November 2004, all cows diagnosed with any type of LDA and viable for surgical treatment from a productive point of view were included in the study. The LDA diagnostic method was based on anamnesis, systematic medical examination, auscultation of the "ping" sound in the left side with percussion, and succussion, detecting the difference with rumen sounds (Rosenberger, 1994). In most cases, the abomasum reached a position between the 9th and the 13th rib on the left side. The time interval between detection of the sick cow by the farmer and surgery was 24 to $48 \mathrm{~h}$. All diagnoses and surgeries were performed by the same veterinarian. A total of 258 cows were in the postpartum period, and 10 cows $(3.73 \%)$ were pregnant between 3 to 8 mo of gestation.

Before surgery, cows were cast and rolled without sedation, following the casting technique outlined by St Jean et al. (1989). In brief, a rope was passed around 


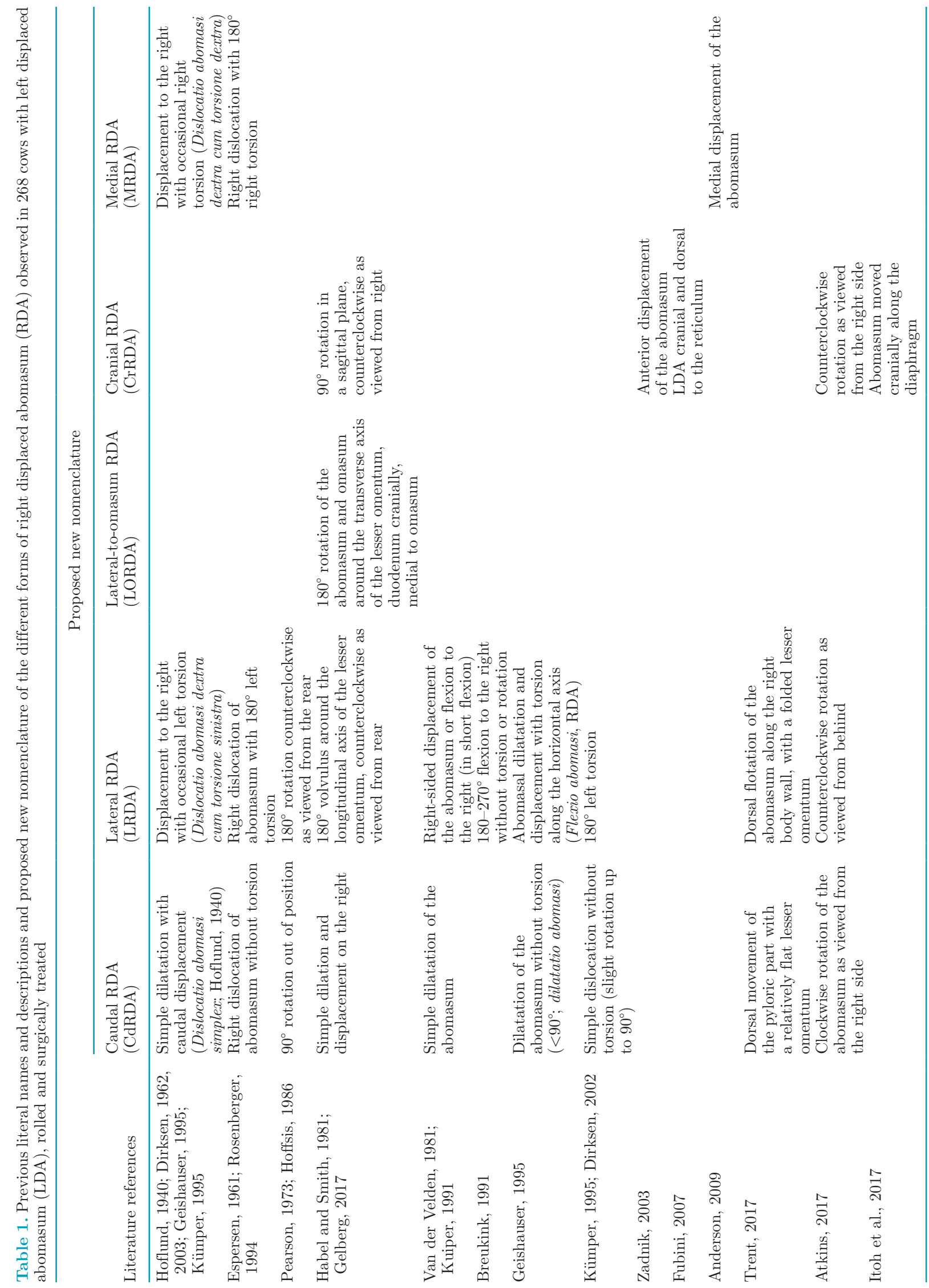


the cow's neck, thorax, and abdomen. Pulling on the rope made cows fall onto their right side. With front and rear legs tied separately, the cows were rolled to dorsal recumbency and then to lateral recumbency on their left side. During the rolling procedure, the gasfilled abomasum was impelled toward the right side of the cow; the position of the abomasum was verified by percussion and simultaneous auscultation throughout the process, in dorsal recumbency and then again in left lateral recumbency. The abdomen was gently knocked or balloted to impel the abomasum into the desired position during every step. In dorsal recumbency, there was no time for the abomasum to naturally evacuate gas, and no percutaneous deflation of the abomasum was attempted, to induce RDA. Once the abomasum was found to be located in the right side, the cows were allowed to stand up by themselves. Normally the whole process was carried out very quickly, within $10 \mathrm{~min}$.

Immediately after rolling, all cows were operated on. Only animals with concurrent diseases were medically treated before surgery (ketosis treatment: intravenous glucose and oral propylene glycol; infectious metritis treatment: parenteral penicillin-streptomycin and flunixin meglumine). Right paralumbar fossa omentopexy was performed as previously described (Trent, 1990). Sedation and anesthesia were performed by epidural injection of $1 \%$ romifidine hydrochloride $0.4 \mathrm{~mL} / 500 \mathrm{~kg}$ plus $2 \%$ lidocaine hydrochloride up to a total volume of $5 \mathrm{~mL}$; local anesthesia was $80 \mathrm{~mL}$ of $2 \%$ lidocaine subcutaneously injected at the incision line. A 15 - to $20-\mathrm{cm}$ incision was performed in the paralumbar fossa, 8 to $10 \mathrm{~cm}$ caudal to the last rib and 8 to $10 \mathrm{~cm}$ below the transverse lumbar processes. Before, during, and after omentopexy, the abdominal cavity was explored through visual inspection and palpation, and the different abomasum positions were systematically recorded. Finally, the routine omentopexy was performed as previously described (Trent, 1990).

Adverse events suspected to be due to the rolling procedure were as follows: 3 cases of mesenteric root volvulus ( 2 cases resolved during surgery, 1 cow died); 3 cases of cecum dislocation (all cases resolved during surgery); 2 cases of cecal retroflexion or cecocolic volvulus; 1 case of cecum torsion; 2 cases of uterus torsion (2 out of 10 pregnant cows, both resolved during surgery. A postsurgical complication observed was 1 abortion (1 of 10 pregnant cows) in the week after surgery, although the cause-effect of the abortion cannot be absolutely demonstrated.

\section{RESULTS AND DISCUSSION}

Immediately after rolling a total of 268 LDA cows, we observed different features regarding the new position of the abomasum, recording the incidence of each RDA type found. We also recorded all postsurgical events and complications.

A limitation of the current study is the external validity of our results. We hypothesize that what we describe reflects the process that can happen in real clinical cases of RDA on farms. In fact, that the RDA can be a further movement after an LDA has already been postulated (Poulsen, 1974). Therefore, the present authors are convinced that these results could be used to infer the actual movements of the RDA in the very early moments of the RDA or AV process. However, we cannot be absolutely confident of this, given that real cases usually show great abomasal dilations, which are allowed to move to the final positions.

\section{RDA and AV Features Observed}

Final abomasum positions found after rolling and surgically treating 268 cows with LDA, and the new proposed nomenclature for each position observed, are as follows.

Abomasum Remained on Left Side. In $1.5 \%$ of all cases (4 out of 268), the abomasum remained on the left side (Figure 1B). In 2 cases this was due to adhesions. In 1 case it was due to the retention of the abomasum by the hepatic ligament, and in 1 case apparent cause could not be identified.

Abomasum Moved to Normal Position. In $54.1 \%$ of cases (145 of 268), the abomasum moved to a normal position. The fundus of the abomasum was located to the left of the midline, and the pyloric region was located on the right side of the abdominal cavity 10 to $30 \mathrm{~cm}$ cranial to the udder base (Wittek et al., 2005a; Figure 1A).

Rolling is a method whereby a cow's position is changed from lateral to supine while the abdomen is pressed with force, for the repositioning of the displaced abomasum from the left side to the normal position (Begg and Witherford, 1956). After rolling, the abomasum returns to its normal position in more than 90\% of cows with LDA, according to previous studies. However, this cannot be described as LDA treatment, due to the high rate of recurrence observed (Uyanik et al., 1982; Hoffsis, 1986). In our study, the percentage of cases with the abomasum observed to return to the normal position $(54.1 \%)$ is far below that reported in previous studies. The reason for this difference could be that we did not try to resolve LDA only by rolling the cow. Instead, as previously noted in Materials and Methods, we tried to move the abomasum completely to the right side, to immediately operate on the cow, record the position of the abomasum, correct the abomasal location if necessary, and perform omentopexy. 


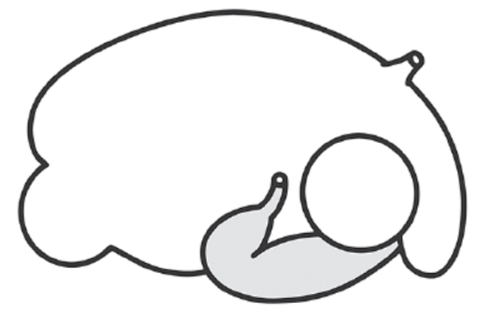

A: Normal position

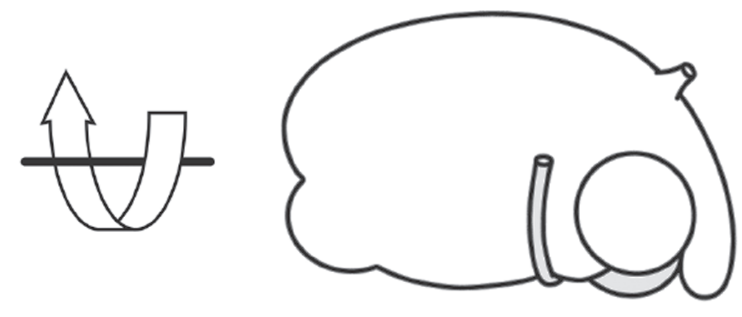

B: LDA

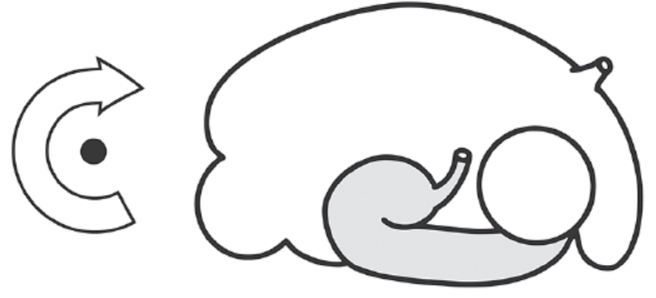

C. $45^{\circ}$ CdRDA

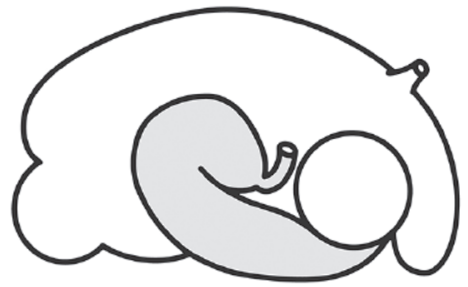

D: $90^{\circ} \mathrm{CdRDA}$

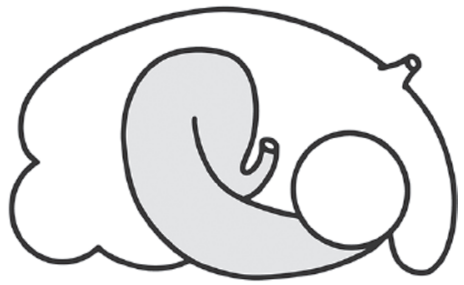

$\mathrm{E}: 180^{\circ} \mathrm{CdRDA}$
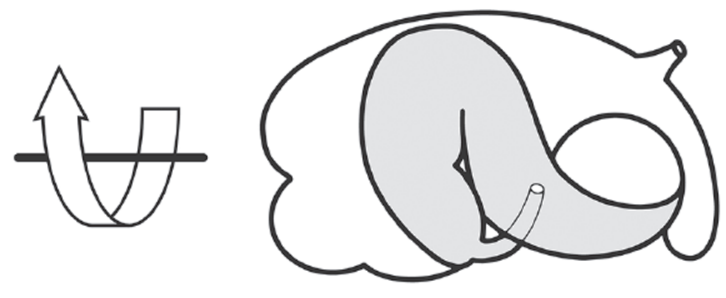

F: $180^{\circ}$ LRDA

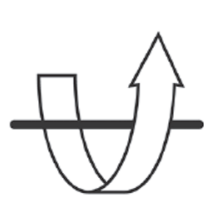

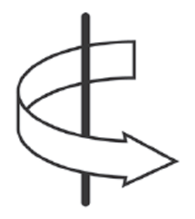

G: AV
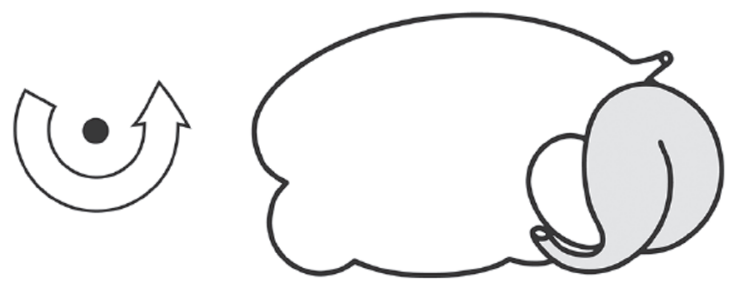

I: CrRDA
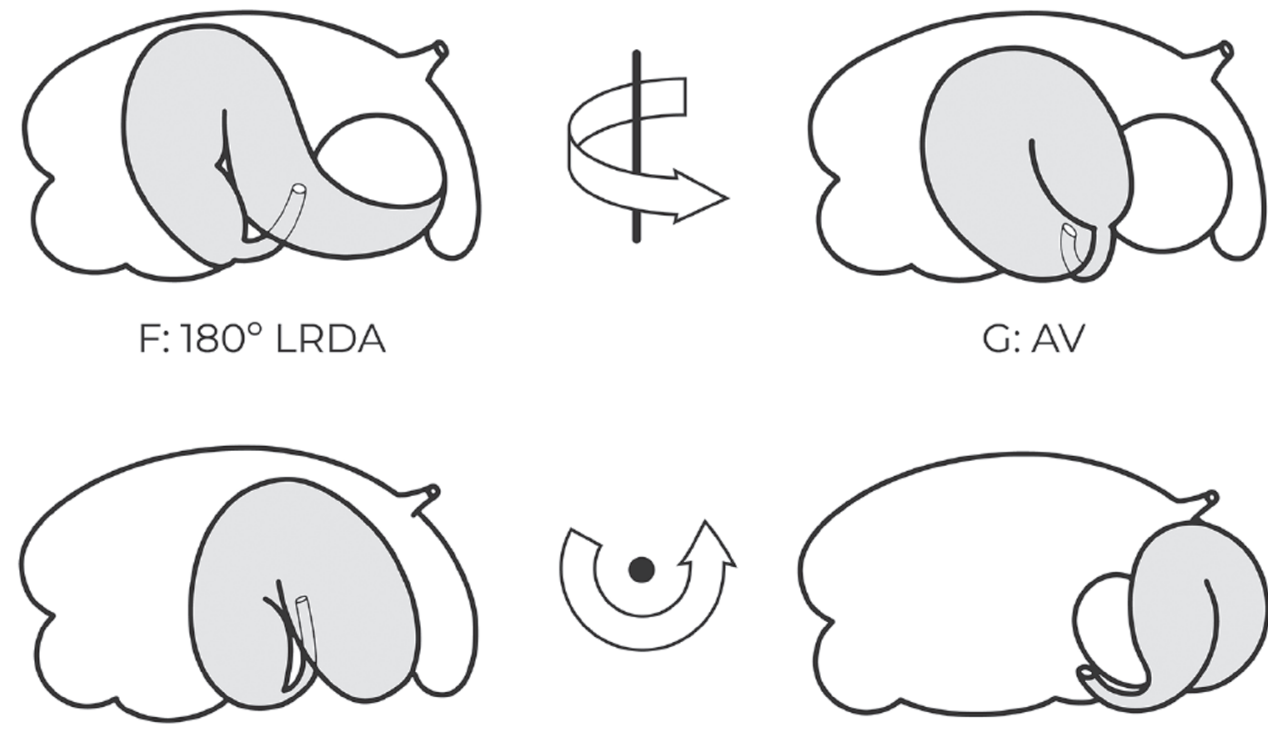

H: LORDA

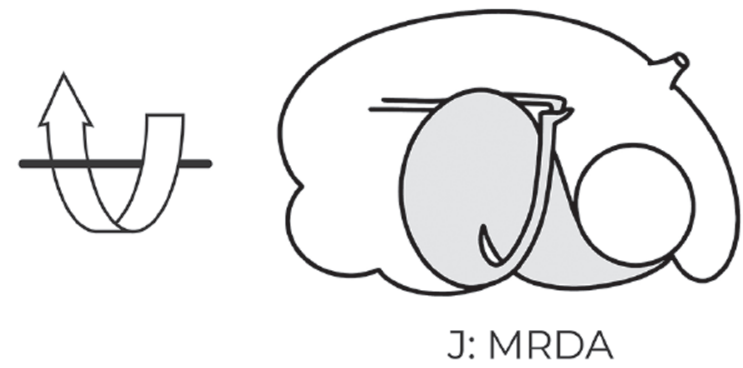

Figure 1. Abomasal positions observed after rolling 268 cows with left displacement of the abomasum (LDA). (A) Normal position. (B) LDA. (C) $45^{\circ}$ caudal right displaced abomasum (CdRDA). (D) $90^{\circ}$ CdRDA. (E) $180^{\circ}$ CdRDA. (F) $180^{\circ}$ lateral right displaced abomasum (LRDA). (G) Abomasal volvulus (AV). (H) Lateral-to-omasum right displaced abomasum (LORDA). (I) Cranial right displaced abomasum (CrRDA). (J) Medial right displaced abomasum (MRDA). 
Abomasum Moved to Right Side. In $44.4 \%$ of cases (119 of 268; Table 2), the abomasum moved to the right side, leading to RDA. Poulsen (1974) was the first to describe RDA as a further movement of an LDA. We could not find in the literature any other incidence rate given for such a complication after rolling cows with LDA, although other features (recovery, relapse of LDA, or AV) have been previously described (Uyanik et al., 1982; St. Jean et al., 1989). This may be due to the speed of the rolling method in our procedure and to the instability of this form of RDA. When rolling cows slowly, 2 events may occur: when the pylorus allows the passage of gas quickly enough, the RDA will deflate, leading to recovery; or, if the pylorus does not allow the RDA to empty the increasing accumulation of gas, this will lead to an AV later. However, we do not have scientific evidence on this hypothesis, and other factors, such as the passage of gas through the omasal canal, could also change the outcome of an initial RDA.

In our study, after rolling promptly and letting the cow stand up immediately, it is likely that we did not allow time for these hypothetical events to occur. This may have led to the higher incidence of RDA observed in our study. If we had allowed gas to pass through the pylorus or the omasal canal, or if gas had been emptied from the abomasum through a cannula (as is the case in the toggle technique), the abomasum would probably have located in its normal position.

Caudal RDA. In cases of RDA following rolling, $58.8 \%$ (70 of $119 ; 26.1 \%$ of the 268 total cows) the abomasum rotated clockwise (from right) from the normal position up to a maximum of $180^{\circ}$, with the greater curvature locating caudally, the lesser curvature locat- ing cranially, the pyloric antrum locating dorsally, and the pylorus locating near the gallbladder. We propose the name caudal RDA (CdRDA) for this type of RDA.

The observed viscera positions found immediately after opening the surgery wound were as follows. In $37.15 \%$ of cases (26 of 70 ), mild distension moved the pylorus below the surgical wound, rising up to $45^{\circ}$ from the normal position (Figure 1C). In $25.7 \%$ of cases (18 of 70 ), moderate distension raised the pylorus $90^{\circ}$, located immediately behind the surgical wound (Figure 1D). In $37.15 \%$ of cases (26 of 70 ), severe distension located the pylorus near the gallbladder, having rotated $180^{\circ}$ from its normal position. This last was the broadest possible rotation in this type of RDA (Figure 1E). Indeed, in such a case, the pyloric antrum makes a greater displacement than the pylorus itself.

We hypothesize that CdRDA occurs when the initial or the most dilated portion of the abomasum is the pyloric part. Severely enlarged abomasum, when slight pressure was applied with the palm of the hand on the dorsal part of the abomasum, suddenly rotated $180^{\circ}$, adopting another form of RDA, described in the following section.

Lateral RDA. In $26.9 \%$ of RDA cases (32 of 119 ; $11.9 \%$ of all cases), the abomasum rotated counterclockwise (from rear) from the normal position up to $180^{\circ}$, caudally to the omasum, with the greater curvature locating dorsally and the lesser curvature and the pylorus ventrally. We propose terming this lateral RDA (LRDA).

The viscera positions observed immediately after opening the surgery wound were as follows. In $28.1 \%$ of LRDA cases (9 of 32), in a moderate displacement,

Table 2. Features observed in 268 cows with left displaced abomasum after being rolled and surgically treated ${ }^{1}$

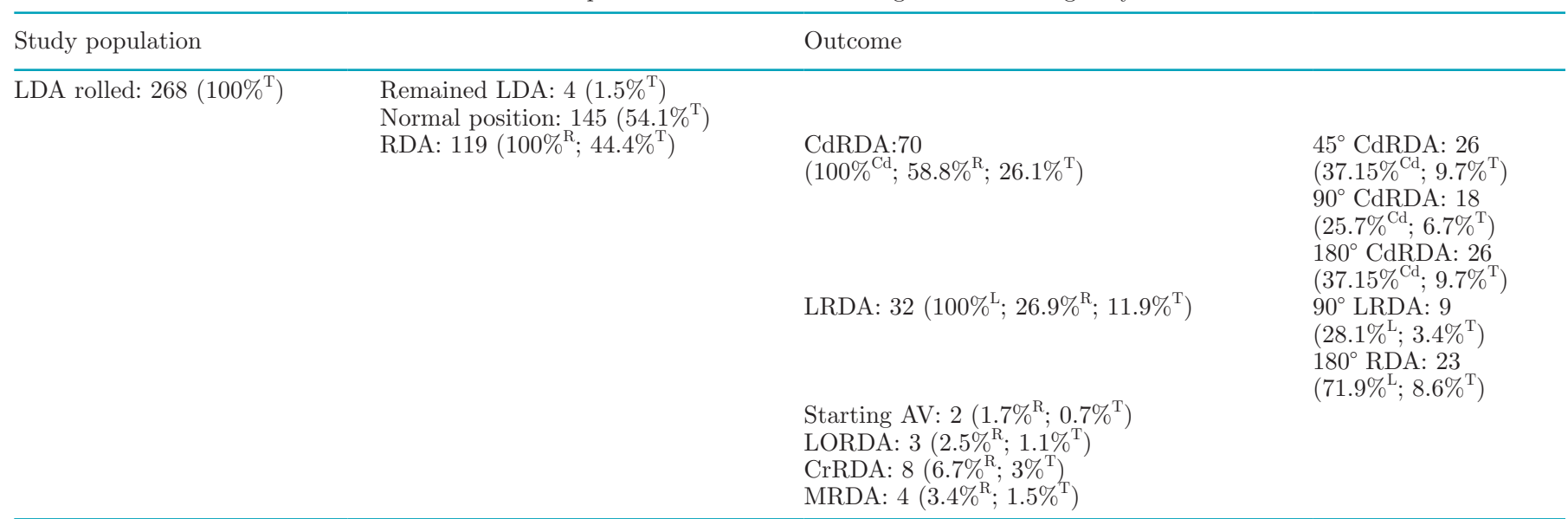

${ }^{1} \mathrm{LDA}=$ left displaced abomasum; RDA $=$ right displaced abomasum; CdRDA $=$ caudal right displaced abomasum; LRDA $=$ lateral right displaced abomasum; $\mathrm{AV}=$ abomasal volvulus; LORDA = lateral-to-omasum right displaced abomasum; CrRDA = cranial right displaced abomasum; MRDA = medial right displaced abomasum. Numbers not in parentheses are total number with the specified outcome. Numbers in parentheses are as follows: $\%^{\mathrm{T}}=$ percentage of the total of rolled LDA cases; $\%^{\mathrm{R}}=$ percentage of $\mathrm{RDA}$ cases; $\%^{\mathrm{Cd}}=$ percentage of CdRDA cases; $\%^{\mathrm{L}}=$ percentage of LRDA cases. 
the abomasum revolved $90^{\circ}$ in a horizontal position, with the greater curvature locating in a lateral position and the lesser curvature in a medial position. In $71.9 \%$ of LRDA cases (23 of 32), the abomasum, in a severe displacement, rotated $180^{\circ}$, with the greater curvature locating in a dorsal position and the lesser curvature and the pylorus ventrally (Figure $1 \mathrm{~F}$ ). In this type of $\mathrm{RDA}, 180^{\circ}$ is probably the maximal rotation possible (we did not see a greater rotation), because, when the intra-abomasal pressure increases further, the abomasum begins to rotate on a different axis, as described in the next section $\left(180^{\circ}\right.$ counterclockwise, from above, initiating the development of an AV).

When we relieved the gas in LRDA, the abomasum returned to CdRDA form, as previously described. We thus consider that LRDA is generally consecutive to CdRDA. However, in some cases, where the first and most dilated portion of the abomasum is the body of the abomasum, LRDA could be the first RDA form.

Abomasal Volvulus. In 2 animals, $1.7 \%$ of the RDA cases ( 2 of $119 ; 0.7 \%$ of the total), the RDA first rotated $180^{\circ}$ counterclockwise (from rear) and then $180^{\circ}$ counterclockwise (from above), initiating the development of an AV (Figure 1G). During gas decompression in these 2 cases, we observed a clockwise (from right) rotation of the abomasum. Several authors have described a higher percentage of AV after rolling cows with LDA than that reported in the current study, as, for example, the $6.7 \%$ cited by Uyanik et al. (1982). This is probably due to the fact that all cows in our study were immediately treated surgically after rolling, with no longer than 30 min between rolling and surgery.

Lateral-to-Omasum RDA. In 3 animals, $2.5 \%$ of RDA cases (3 of $119 ; 1.1 \%$ of the total), the abomasum rotated counterclockwise (from rear), laterally to the omasum. The name we propose for this RDA form is lateral-to-omasum RDA (LORDA; Figure 1H). This kind of RDA has been described only rarely (Habel and Smith, 1981; Gelberg, 2017), which tallies with the low incidence found in the current study.

Cranial $R D A$. In $6.7 \%$ of cases (8 of $119 ; 3 \%$ of the total), the abomasum rotated counterclockwise (from right), cranially to the omasum. We propose the name cranial RDA (CrRDA) for this form (Figure 1I). Most authors have described CrRDA as very rare (Neal and Pinsent, 1960; Fubini, 2007; Gelberg, 2017), except Zadnik (2003), who cited an incidence of $12.4 \%$ of treated displaced abomasum cases. Recently, Itoh et al. (2017), using fluoroscopy after rolling 12 LDA cows, observed how the abomasum moved in a cranial direction along the diaphragm in 5 cases. Three of these cases led to LDA, and the other 2 cows presented with RDA. The rare clockwise turn of the AV (Kümper, 1995) was not observed in the current study.
Medial RDA. In $3.4 \%$ of RDA cases (4 of $119 ; 1.5 \%$ of the total), the abomasum rotated $180^{\circ}$ clockwise (from rear) and located between the greater omentum and the rumen, with the greater curvature ending dorsally and the lesser curvature ventrally (similar to the position of an LDA but located medially to the rumen instead of laterally). We propose the name medial RDA (MRDA) for this extremely rare type of displacement (Figure 1J).

This form could match the type described by other authors as "torsion of abomasum to the right" (Dirksen, 1962; Habel and Smith, 1981; Kümper, 1995). More recently, Anderson (2009) cited just 3 cases of "medial displacement of the abomasum" over 10 years of clinical practice. Correspondingly, in our daily practice, we also have rarely observed MRDA cases.

\section{Adverse Events and Postsurgical Complications}

In our study, the relapse rate of displaced abomasum after rolling and surgery was $1.5 \%$ (4 of 268), which is consistent with the $2.0 \%$ observed by Sexton et al. (2007). In the studied LDA cows, total postsurgical mortality after rolling was $1.86 \%$ ( 5 of 268 ): 2 cases of preoperative peritonitis leading to death; 1 case of aspiration pneumonia; 1 case of clostridial myositis after an intramuscular calcium solution infusion; and 1 case of inoperable mesenteric root volvulus due to the rolling procedure, immediately resulting in death.

As percentages, $0.75 \%$ (2 of 268) of abomasal adhesions and $0.75 \%$ (2 of 268 ) of deadly preoperative peritonitis are lower than those reported previously in LDA cases using the same surgical technique as ours: $3.7 \%$ for adhesions and 1.9\% for mortality (Sexton et al., 2007). There may be several reasons for this, such as our clinic's short time between diagnosis and surgery.

Regarding adverse events observed and postsurgical complications, mesenteric volvulus after rolling has been widely cited (Kelton et al., 1988; Opsomer et al., 1998) but usually as an extraordinary case report. Although the possibility of uterine torsion being caused by rolling cows in the last third of pregnancy has been suggested (Braun, 1968; St. Jean et al., 1989), no actual cases have been reported.

\section{RDA Etiopathogenesis Hypothesis}

Based on our observations in this study, the authors propose a description of the probable ethiopathogenesis and consecutive movements of the RDA from the very early phases until AV development (Figure 1). First, for a better understanding, we summarize our proposed nomenclature of the different RDA types in Table 1, matching all previously described RDA positions with 
their literal descriptions and our suggested nomenclature.

Typically, the ratio of RDA to AV is around 1 to 9 (Kümper, 1995), although other authors have reported very similar incidences of RDA and $\mathrm{AV}$ (47.6\% and $52.4 \%$, respectively; Fubini et al., 1991). However, in our study, most abomasal displacements were RDA (RDA: 98.3\%; AV: 1.7\%). This may be due to the fact that the cows were operated on immediately after rolling, such that they did not have time to develop AV, our aim being to observe the very early moments of RDA developing from LDA. Most published studies are on sick animals with advanced displaced abomasum disease. Similarly, in the clinical authors' experience, the majority of cases of RDA operated on are AV. We speculate that cases of RDA probably do not induce severe clinical signs until they evolve to AV.

In our study, simple dilatation with caudal displacement was the most common type of RDA observed, representing $58.8 \%$ of all cases. Of these caudal displacements, $37 \%$ rotated $45^{\circ}, 26 \%$ rotated $90^{\circ}$, and $37 \%$ rotated $180^{\circ}$. This kind of RDA, which we term CdRDA, has been widely cited by other authors with different degrees of rotation (Table 1; Neal and Pinsent, 1960; Geishauser, 1995; Gelberg, 2017). In our study, the maximal CdRDA rotation observed was $180^{\circ}$ clockwise (from the right side of the cow), with the pylorus located close to the gallbladder and the antrum pyloricum dorsal to the pylorus. In contrast, Dirksen (2002) states that the maximum turning of such an RDA can only be $90^{\circ}$. This contradiction may be due to a difference in the anatomic part of the abomasum used in describing the degree of rotation, since the pylorus actually moves only slightly (probably $90^{\circ}$ ), although the pyloric part of the abomasum rotates $180^{\circ}$.

These movements are an evolution of the CdRDA, progressing to a broader rotation depending on other factors, most probably the amount of gas inside the abomasum. In fact, when we released the gas from the $180^{\circ}$ CdRDA abomasum with a needle, the distended abomasum rotated counterclockwise (from lateral), inducing first a $90^{\circ} \mathrm{CdRDA}$, then a $45^{\circ} \mathrm{CdRDA}$, and finally, when completely deflated, the abomasum located in its normal position.

In cases of extreme dilation of a CdRDA, we have observed that with slight downward pressure exerted by the palm of the hand on the pyloric part of the abomasum, the RDA suddenly moves and becomes, according to our suggested nomenclature, an LRDA, the second most common RDA type in our study (32 of 119). A total of 9 LRDA cases rotated $90^{\circ}$, and 23 rotated $180^{\circ}$. The LRDA form has also been widely described under other names in the literature (Table 1; Dirksen, 1962;
Geishauser, 1995; Constable et al., 2017). In the case of a $180^{\circ}$ rotated LRDA, when pressure is released with a needle, the abomasum rotates clockwise (from rear), rotates back to a $90^{\circ} \mathrm{LRDA}$, and finally rotates back until it reaches the normal position.

In our study, $28.1 \%$ of the animals (9 of 32 ) showed an abomasum rotated $90^{\circ}$ in a horizontal position, with the greater curvature locating in a lateral position and the lesser curvature in a medial position. This situation could be (1) an early phase of the $180^{\circ}$ LRDA or (2) a $180^{\circ}$ LRDA that has begun to lose gas. This latter option is, in our opinion, the most likely, because we were able to see the proximal and descendent duodenum in a parallel position and completely distended, which indicates that gas was entering the duodenum. However, in some cases, when releasing the gas, the LRDA moved abruptly and became a CdRDA.

Furthermore, it is said that to correct an abomasal volvulus, the abomasum must first be "turned back" to an LRDA; then, the LRDA must be turned back to a CdRDA; and finally, the CdRDA must be turned back to the normal abomasum position (Dirksen, 2002). In the normal position of the abomasum, when a cow lies on its right side, the uppermost point of the abomasum is the large curvature of the abomasum body, which triggers the LDA. When the cow lies on its left side, the uppermost point of the abomasum is the pyloric part, hence the most easily dilatable part. Therefore, we suggest that the CdRDA is the first stage in the dilatation and movement of an RDA.

The pyloric part is smaller and less dilatable than the abomasal body. Therefore, when a CdRDA reaches its maximum dilatation and rotation, the increasing pressure will expand the abomasum body, and it will move lateral and dorsally, counterclockwise from the rear. Consequently, we propose that the origin of an LRDA is a severely gas-filled CdRDA, with the LRDA being the second stage in the pathogenesis of the RDA.

In our study, 2 cases $(1.7 \%)$ had initiated the formation of an abomasal volvulus rotating $180^{\circ}$ counterclockwise (from above) from an initial $180^{\circ}$ LRDA. The probable reason for this very small percentage of cases with this feature is that we always performed the surgical treatment immediately after rolling the cows. Moreover, when decompressing the AV, a clockwise (from right and above) rotation was observed in both animals. This description is in line with Dirksen (1962), Constable et al. (1991, 2017), and Kümper (1995). Therefore, LRDA may be the second stage in the dilatation and movement of an RDA and the last stage before an AV.

Two much less frequent observations in our study were the abomasum located between the omasum 


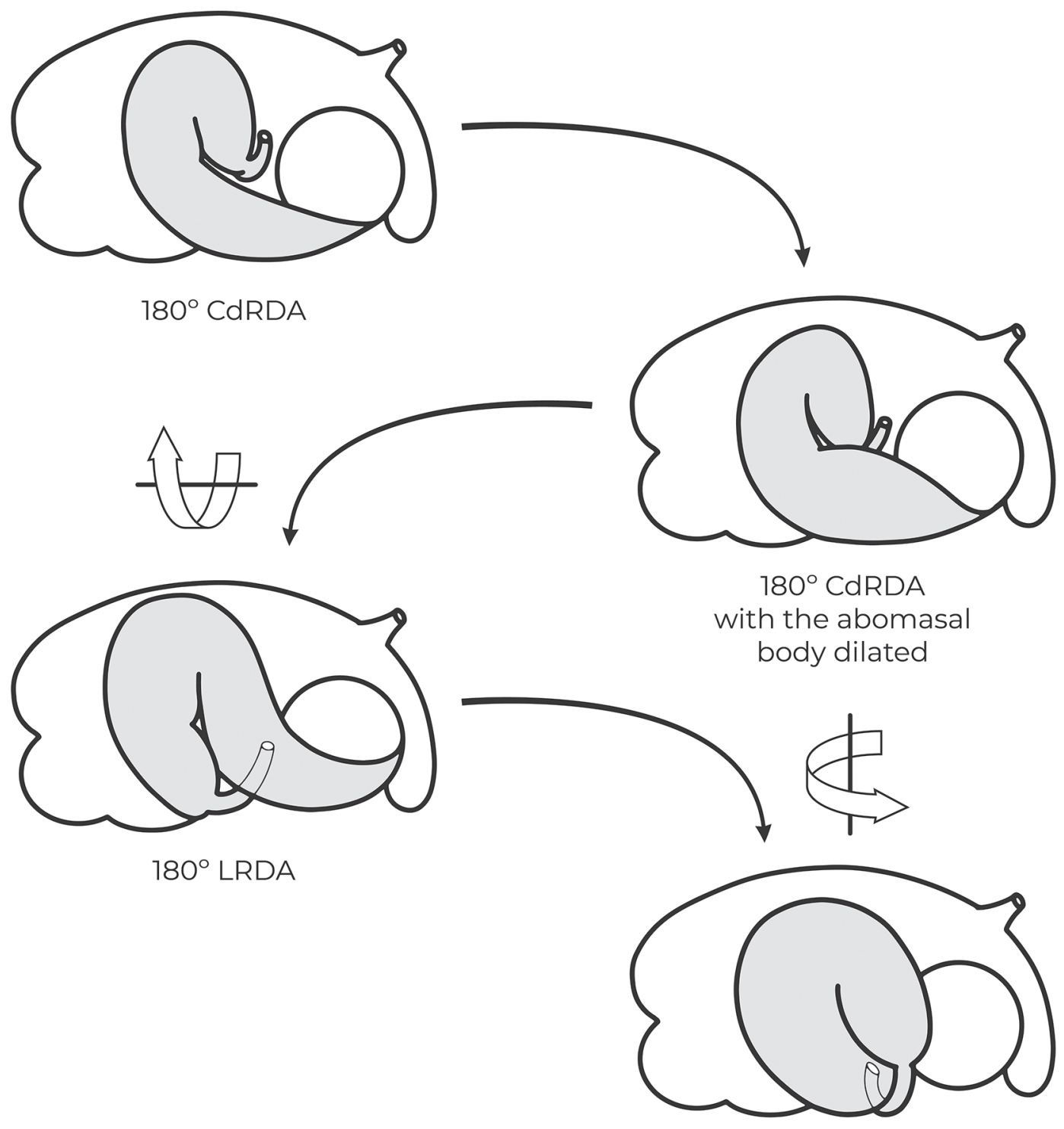

AV

Figure 2. Formation of an abomasal volvulus (AV) from an initial $180^{\circ}$ caudal right displaced abomasum (CdRDA) that first dilates the abomasal body, then rotates $180^{\circ}$ counterclockwise (as viewed from rear) and becomes a lateral right displaced abomasum (LRDA), and finally rotates $180^{\circ}$ counterclockwise (as viewed from above).

and the body wall $(2.5 \%$ of cases), which we named LORDA, and the abomasum rotated cranially to the omasum (6.7\% of cases), which we named CrRDA. These positions may be the result of the movements of a CdRDA in the case of cows with empty prestomachs that lie down on a sloped floor with the front part of their bodies in a higher position than the rear part. Another possibility may be cows standing up on their front legs first, although this is very rare. The LORDA and CrRDA types of RDA are probably the stages before an OAV or ROAV, respectively, if gas filling increases.
These movements have been partially cited by Habel and Smith (1981), Gelberg (2017), and Atkins (2017).

Finally, we observed an abomasum located between the greater omentum and the rumen, with the greater curvature in dorsal position and the lesser curvature in ventral position (3.4\% of cases), which we have named MRDA. This extremely rare condition could be caused in the same way as LDA but with the large curvature of the abomasum placed close to the medial wall of the rumen at the beginning of the gas filling, facilitated by an empty rumen at the initiation of the illness. Addition- 


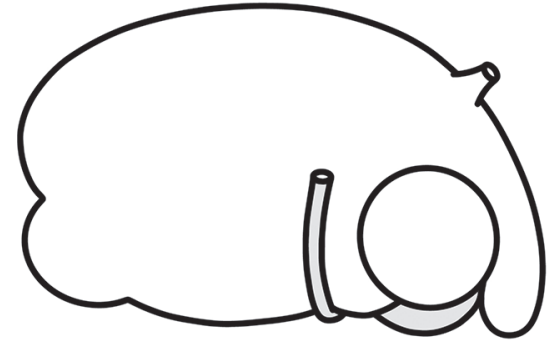

B: LDA

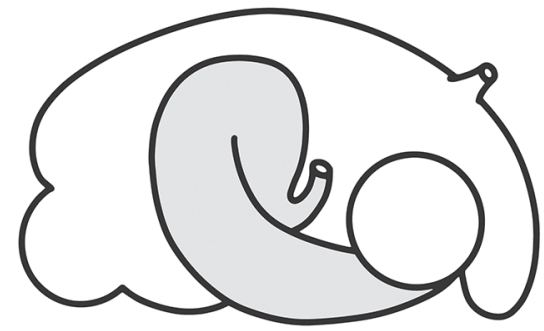

E: $180^{\circ} \mathrm{CdRDA}$

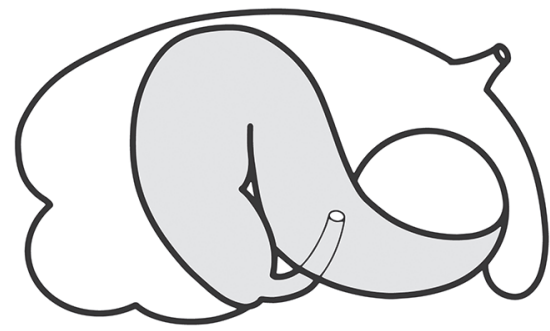

F: $180^{\circ}$ LRDA
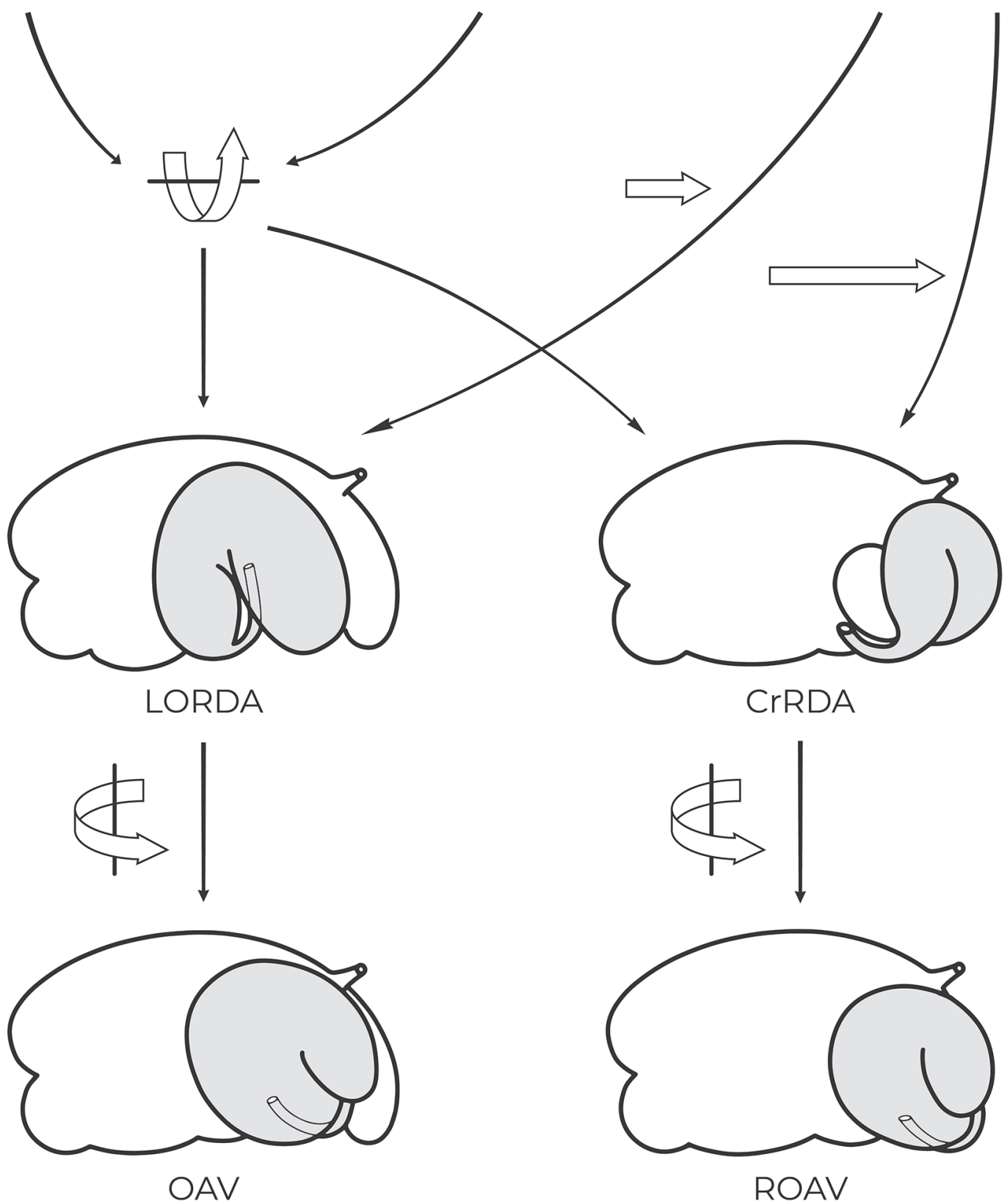

Figure 3. Left displaced abomasum (LDA; see Figure 1B) and caudal right displaced abomasum (CdRDA; Figure 1E) rotating counterclockwise (as viewed from rear), and lateral right displaced abomasum (LRDA) rotating cranially (Figure 1F) could be the initial forms of the lateral-to-omasum right displaced abomasum (LORDA) and cranial right displaced abomasum (CrRDA), respectively. LORDA and CrRDA can lead to an omasum-abomasum volvulus (OAV) or to a reticulum-omasum-abomasum volvulus (ROAV), respectively. 
ally, we had previously observed MRDA in 2 cases (our unpublished data) and, without rolling, successfully surgically treated the cows. Our description matches 3 other cases reported previously (Anderson, 2009).

Regarding the clinical effects of the different positions of RDA, it is worth discussing the fact that several RDA features described here, despite being abnormal (not physiological) abomasal positions, can be consistent with subclinical disease and may not induce any serious production impairment, as previously described for the LDA (Fubini, 2007). In our opinion, as long as digestive transit and the digestive blood supply are maintained, no clinical disturbance may be observed, although the condition will probably decrease the rate of abomasal emptying, as observed in LDA cows (Wittek et al., 2005b). However, these abnormal RDA positions are unstable and can shift rapidly into very severe forms, such as AV, or into the pathological condition of an LDA (Itoh et al., 2017). Therefore, we are convinced of the appropriateness of the medical indication for surgical treatment of LDA and RDA cows. Although an excessively caudo-dorsal pyloric omentopexy or toggle pin fixation could induce an RDA (and indeed, it is a risk of this surgical treatment), the most threatening form, $\mathrm{AV}$, is no longer possible.

In brief, in accordance with our observations, we propose that the full sequence of the different forms of RDA, from the initial LDA up to AV, is as follows:

- CdRDA becomes an LRDA with increasing pressure (Figure 2)

- LRDA becomes an AV when rotated counterclockwise from above (Figure 2)

- LDA, CdRDA, and LRDA could be the initial forms of LORDA or CrRDA, which occur when the forestomachs are empty or when the animal moves in a specific way (e.g., when it stands up on its front legs first; Figure 3)

- LORDA and CrRDA forms can lead to OAV or, in extreme cases, to ROAV when pressure increases (Figure 3)

In our opinion, factors that cause these different types of RDA and the different sequences may include severity of dilatation and amount of gas, liquid, feed, and sand in the abomasum; size of the cow's abdominal cavity (corporal depth); level of rumen fill; possibility of gas moving from the abomasum through the pylorus; gas passing from the duodenum to the jejunum; degree of previous engorgement of the different parts of the duodenum; and inclination of the ground and the way in which the cow lies down and stands up.

\section{CONCLUSIONS}

With this study we have been able to establish, for the first time, the sequence and incidence of each RDA location and its complications. This new knowledge will allow us to accurately assess and correct this highly prevalent bovine disease.

\section{ACKNOWLEDGMENTS}

The authors thank Francisco Velasco for his help in the design of the figures.

\section{REFERENCES}

Anderson, D. E. 2009. Pathophysiology of displacement of the abomasum in cattle. Pages 35-40 in Current Veterinary Therapy: Food Animal Practice. 5th ed. Elsevier Inc., Amsterdam, the Netherlands.

Atkins, D. G. 2017. Practical approaches to on-farm bovine surgery. Page 37 in Proc. Central Canadian Veterinary Conference, Winnipeg, Manitoba.

Begg, H., and W. A. Witherford. 1956. Displacement of the abomasums in the cow. Vet. Rec. 68:122-125.

Braun, R. K. 1968. Non-surgical correction of LDA in cow. Cornell Vet. 58:111-116.

Breukink, H. J. 1991. Abomasal displacement, etiology, pathogenesis, treatment and prevention. Bov. Pract. 26:148-156.

Constable, P. D., K. W. Hinchcliff, S. H. Done, and W. Grünberg. 2017. Diseases of the abomasum. Pages 501-523 in Veterinary Medicine: A Textbook of the Diseases of Cattle, Horses, Sheep, Pigs, and Goats. 11th ed. Elsevier, St Louis, MO.

Constable, P. D., G. St. Jean, B. L. Hull, D. M. Rings, and G. F Hoffsis. 1991. Prognostic value of surgical and postoperative findings in cattle with abomasal volvulus. J. Am. Vet. Med. Assoc. 199:892-898.

Dirksen, G. 1962. Die Erweiterung, Verlagerung und Drehung des Labmagens beim Rind. Tierärztliche Hochschule, Habilitation, Hannover, Germany.

Dirksen, G. 2002. Krankheiten der Verdauungsorgane in der Bauchwand. Krankheiten des Darmes. Pages 473-514 in Innere Medizin und Chirurgie des Rindes. 4th ed. G. Dirksen, H.-D. Gründer, and M. Stöber, ed. Blackwell Wissenschafts-Verlag und Parey Buchverlag, Berlin, Germany.

Espersen, G. 1961. Die rechtsseitige Labmagenerweiterung und -verlagerung beim Rind. Dtsch. Tierarztl. Wochenschr. 68:2-7.

Fubini, S. L. 2007. Noninfectious diseases of the gastrointestinal tract. Pages 130-199 in Rebhun's Diseases of Dairy Cattle. 2nd ed. T. J. Divers and S. F. Peek, ed. Saunders Elsevier, St. Louis, MO.

Fubini, S. L., Y. T. Grohn, and D. F. Smith. 1991. Right displacement of the abomasum and abomasal volvulus in dairy cows: 458 cases (1980-1987). J. Am. Vet. Med. Assoc. 198:460-464.

Geishauser, T. 1995. Abomasal displacement in the bovine - A review on character, occurrence, aetiology and pathogenesis. Zentralbl. Veterinarmed. A 42:229-251.

Gelberg, H. B. 2017. Alimentary system and the peritoneum, omentum, mesentery and peritoneal cavity. In Pathologic Basis of Veterinary Disease Expert Consult. 6th ed. J. Zachary, ed. Elsevier, Amsterdam, the Netherlands.

Habel, R. E., and D. F. Smith. 1981. Volvulus of the bovine abomasum and omasum. J. Am. Vet. Med. Assoc. 179:447-455.

Hoffsis, G. F. 1986. Diseases of the abomasum and intestinal track. In Current Veterinary Therapy. 2nd ed. J. L. Howard, ed. W. B Saunders, Philadelphia, PA. 
Hoflund, S. 1940. Untersuchungen über Störungen in den Funktionen der Wiederkäuermagen, durch Schädigungen des Nervus vagus verursacht. Svensk. Vet. Tidskr. 45(Supplement):322-326.

Itoh, M., T. Aoki, Y. Sakurai, N. Sasaki, H. Inokuma, S. Kawamoto, and K. Yamada. 2017. Fluoroscopic observation of the development of displaced abomasum in dairy cows. J. Vet. Med. Sci. 79:1952-1956. https://doi.org/10.1292/jvms.16-0611.

Kelton, D. F., J. García, C. L. Guard, R. P. Dinsmore, P. M. Powers, M. C. Smith, S. Stelman, N. Ralston, and M. E. White. 1988. Bar suture (toggle pin) vs. open surgical abomasopexy for treatment of left displaced abomasum in dairy cattle. J. Am. Vet. Med. Assoc. 193:557-559.

Kuiper, R. 1991. Abomasal disorders. Bov. Pract. 26:111-116.

Kümper, H. 1995. [Right displacement of the abomasum in cattle. 1. Pathogenesis, clinical course and prognosis.] Tierarztl. Prax. 23:351-359.

Neal, P. A., and P. J. N. Pinsent. 1960. Dilatation and torsion of the bovine abomasum. Vet. Rec. 72:175-181.

NRC (National Research Council). 2001. Nutrient Requirements of Dairy Cattle.7th rev. ed. Natl. Acad. Press, Washington, DC.

Opsomer, G., L. Laurier, A. de Kruif, and P. D. Murray. 1998. Left displaced abomasum: Considerations of treatment method and a case report of mesenteric torsion after rolling. Vet. Q. 20:22-24. https://doi.org/10.1080/01652176.1998.9694830.

Pearson, H. 1973. The treatment of surgical disorders of the bovine abdomen. Vet. Rec. 92:245-254.

Poulsen, J. S. D. 1974. Clinical chemical examination of a case of left sided abomasal displacement changing to right sided abomasal displacement. Nord. Vet. Med. 26:91-96.

Rosenberger, G. 1994. Pages 351-361 in Krankheiten des Rindes. 3rd ed. Blackwell Wissenschafts-Verlag und Parey Buchverlag. Berlin, Germany.
Sexton, M., W. Buckley, and E. Ryan. 2007. A study of 54 cases of left displacement of the abomasum: February to July 2005. Ir. Vet. J. 60:605-609. https://doi.org/10.1186/2046-0481-60-10-605.

St Jean, G., P. D. Constable, B. L. Hull, and D. M. Rings. 1989. Abomasal volvulus in cattle following correction of left displacement by casting and rolling. Cornell Vet. 79:345-351.

Trent, A. M. 1990. Surgery of the bovine abomasum. Vet. Clin. North Am. Food Anim. Pract. 6:399-448.

Trent, A. M. 2017. Surgery of the abomasum. In Farm Animal Surgery. 2nd ed. S. L. Fubini and N. G. Ducharme, ed. Elsevier, St. Louis, MO.

Uyanik, N., S. Dijk, P. Beukelen, R. Kuiper, and H. J. Breukink. 1982. Ervaringen met de behandeling van de lebmaagdislocatie naar links door middle van de wentelmethode. Tijdschr. Diergeneeskd. 107:259-263.

Van der Velden, N. 1981. De lebmaagdislocatie naar rechts bij het rund. Topografische aspecten. PhD Dissertatie Diiergeneeskunde, Utrecht, the Netherlands.

Wittek, T., P. D. Constable, and D. E. Morin. 2005a. Ultrasonographic assessment of change in abomasal position during the last three months of gestation and first three months of lactation in HolsteinFriesian cows. J. Am. Vet. Med. Assoc. 227:1469-1475.

Wittek, T., K. Schreiber, M. Fürll, and P. D. Constable. 2005b. Use of the $\mathrm{D}$-xylose absorption test to measure abomasal emptying rate in healthy lactating Holstein-Friesian cows and in cows with left displaced abomasum or abomasal volvulus. J. Vet. Intern. Med. 19:905-913.

Zadnik, T. 2003. Review of anterior displacement of the abomasum in cattle in Slovenia. Vet. Rec. 153:24-25. https://doi.org/10.1136/ vr.153.1.24. 